\title{
High intensity pulse self-compression in short hollow core capillaries
}

\author{
Thomas J. Butcher ${ }^{1}$, Patrick N. Anderson ${ }^{1}$, Peter Horak ${ }^{1}$, Jeremy G. Frey ${ }^{2}$, William S. Brocklesby ${ }^{1}$ \\ 1. Optoelectronics Research Centre, University of Southampton, Highfield, Southampton, SO171BJ, UK \\ 2. School of Chemistry, University of Southampton, Highfield, Southampton, SO171BJ, UK
}

The drive for shorter pulses for use in techniques such as high harmonic generation and laser wakefield acceleration requires continual improvement in post-laser pulse compression techniques. The two most commonly used methods of pulse compression for high intensity pulses are hollow capillary compression via self-phase modulation (SPM) [1] and the more recently developed filamentation [2]. Both of these methods can require propagation distances of 1-3 $\mathrm{m}$ to achieve spectral broadening and compression. Additionally, hollow capillary compression requires post compression of the broadened pulse by chirped mirrors. Filamentation trades the efficiency of hollow capillary compression (67\%) for ionisation-induced pulse self-compression. A mixture of SPM and plasma generation increases the spectral bandwidth of the pulse; however this occurs only in a small region at the centre of the beam. Spatial filtering is required to achieve the shortest pulses, reducing the efficiency to $20 \%$. Although the majority of hollow core capillary compression requires long propagation distances, compression in short capillaries [3] with significant plasma generation has been demonstrated to be a promising technique.

Here we present pulse self-compression in a $7 \mathrm{~cm}$ hollow core capillary with a reduction in pulse length from $53 \mathrm{fs}$ to $16 \mathrm{fs}$ shown in figure 1 . The efficiency of this process (37\%) is greater than that of filamentation, whilst maintaining the benefits of pulse self-compression, removing the need for post-capillary compression.
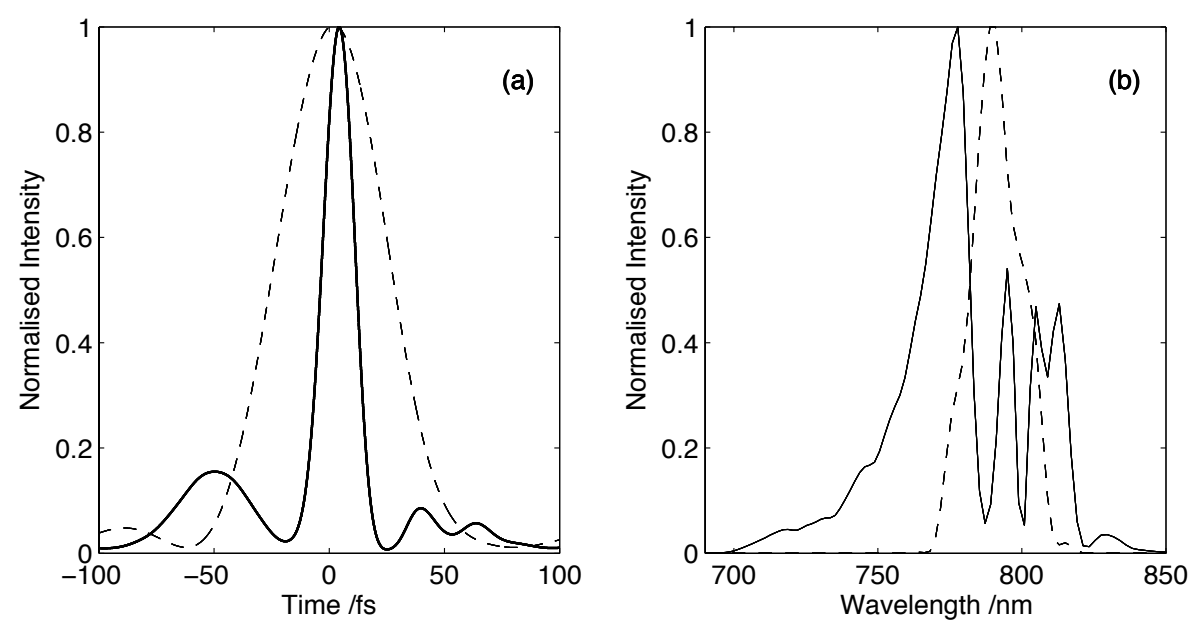

Fig. 1 Comparison of the temporal (a) and spectral (b) distributions of the pulse prior to (dashed) and following (solid) compression within the capillary. The output pulse has a FWHM of $16 \mathrm{fs}$ compared to $53 \mathrm{fs}$ for the input pulse.

A $53 \mathrm{fs}, 600 \mu \mathrm{J}$ pulse centred at $790 \mathrm{~nm}$ was coupled into a $7 \mathrm{~cm}$ hollow core capillary with a core radius of $75 \mu \mathrm{m}$. Argon gas was injected into the capillary through two holes drilled $2 \mathrm{~cm}$ from either end Typical pressures were between 50 and 200 mbar. The time profile of the measured 16 fs output pulse and its corresponding spectrum are shown in figure 1 in comparison with the input pulse. Propagation of the pulse is modelled using a multimode nonlinear Schrödinger equation, including the effects of ionization. The observed spectral broadening due to both SPM and plasma generation matches that predicted by the model. The measured efficiency of this process is $37 \%$.

We have observed similar spectral and temporal changes in capillaries as short as $4.5 \mathrm{~cm}$, with scaleable pressures and powers. Unlike both filamentation and traditional hollow capillary compression the propagation distance required for significant spectral broadening is small, reducing the bench space required. In addition the self-compression of the pulse can save both space and money by removing the need for post compression. Thus we have produced an efficient and compact alternative for compressing high intensity pulses.

\section{References}

[1] S. Bohman, A. Suda, T. Kanai, S. Yamaguchi, K. Midorikawa, "Generation of $5.0 \mathrm{fs}, 5.0 \mathrm{~mJ}$ pulses at $1 \mathrm{kHz}$ using hollow-fiber pulse compression", Opt. Lett. 35, 1887-1889 (2010)

[2] O. Varela, B. Alonso, I. J. Sola, J. San Román, A. Zair, C. Méndez and L. Roso, "Self-compression controlled by the chirp of the input pulse", Opt. Lett. 35, 3649-3651 (2010)

[3] N. L. Wagner, E. A. Gibson, T. Popmintchev, I. P. Christov, M. M. Murnane and H. C. Kapteyn, "Self-Compression of Ultrashort Pulses through Ionization-Induced Spatiotemporal Reshaping”, Phys. Rev. Lett. 93, 173902 (2004) 